Dr. Konrad Kraemer (director of KNA, Bonn), the writer of this article is the president of the German National Committee for the organization of the 8th World Congress of the Catholic Press (UCIP). The main work in preparing this congress was done by the late Fr. Emile Gabel. The theme of the meeting, "World-Church-Press", will be to demonstrate "right order" in human society. The press includes other media of social communication as well; and all Catholic journalists are invited. A review of the basic references and working groups of the congress shows that the main concern will be discussion about practical problems. The congress is to work - as far as this is possible for a Catholic association - in an ecumenical spirit. Berlin was chosen as the site for this congress because of the progressive attitude of the German speaking Catholics at the Second Vatican Council.

\title{
RESUMEN
}

El autor, Dr. Konrad W. Kraemer (KNA, Bonn), ha preparado el Congreso Mundial berlinés de la Prensa Católica (2-6 julio 1968) para el Comité Nacional alemán organizador del Octavo Congreso Mundial de la UCIP. El trabajo principal corrió a cargo del defunto P. Emile Gabel. El tema "Mundo - Iglesia - Prensa" debe ser considerado como un gran esfuerzo en pro de un orden social más justo. "Prensa" representa un concepto más ámplio que incluye también los problemas de los otros medios periodísticos: todos los periodístas católicos de cualquier medio de información deben darse por aludidos. Un vistazo a las ponencias principales y a los círculos de trabajo del Congreso nos descubre que, lo más importante va a ser la meditación y discusión de los problemas prácticos. El Congreso desea trabajar ecuménicamente, en la medida que ésto es posible dentro del cuadro de una Unión Católica Mundial. La elección de Berlín y Alemania como sede del Congreso se debe, en gran parte, al interés despertado por la posición de avanzada que mantuvo el catolić́smo alemán durante el Concilio Vaticano II.

\section{Entwicklungshilfe im Bereich der Massenmedien}

\section{von Otto Kaspar}

„Wer von Euch, der einen Turm bauen will, setzt sich nicht zuerst hin und berechnet die Kosten, ob er genug habe, um fertig zu bauen?" Das ist die klassische Stelle bei Lukas $(14,28)$, die uns die Maßstäbe auch für die Massenmedienhilfe der Kirche liefert. Wenn man dazu noch seinen Appell nimmt, nicht "ohne festen Grund“ zu bauen, so hat man ein fertiges Programm dieser Massenmedienhilfe.

In New York beim Weltkongreß der katholischen Presse 1965 hat eine Resolution aufgezeigt, wie dieses Programm verwirklicht werden soll. Den Auftrag dazu hatte der Papst bei dem vorhergehenden Weltkongreß der katholischen Presse in Santander 1962 gegeben. Durch ein Schreiben des damaligen Staatssekretärs Kardinal Tardini beauftragte er offiziell die UCIP, die Katholische Weltunion der Presse, den Auf- und Ausbau der katholischen Presse in den Entwicklungsländern in die Hand zu nehmen. Der Papst gab damals auch die Schwerpunkte an: „Nicht allein durch die Vermitt-

Dr. Otto Kaspar ist Chefredakteur des Bistumsblattes „Ruhrwort" (Essen) und Vorsitzender der Entwicklungskommission der Katholischen Weltunion der Presse (UCIP). 
lung materieller Hilfe und von Maschinen, die ihnen fehlen, sondern noch mehr durch die Entsendung von Hilfskräften, die technisch qualifiziert und in der Lage sind, Fachkräfte auszubilden und vielleicht auch die Verbreitung einer gesunden Nachrichtengebung $\mathrm{zu}$ fördern. ${ }^{\text {" }}$

In den drei Jahren von Santander bis New York ist UCIP-Generalsekretär Emile Gabel $(\dagger)$ mit aller Energie an diese Aufgabe herangegangen. Niederländische und deutsche Initiativen kamen ihm zu Hilfe. Sie waren notwendig und wichtig, weil in der Kirche das Verständnis für die Massenmedien als solche und insbesondere für jene in den Entwicklungsländern nicht übertrieben groß war.

Durch die Aktion „Adveniat“" wurde ein Durchbruch in der Massenmedienhilfe möglich. Die Bischöfliche Kommission für „Adveniat" hatte bei Pater Gabel eine Untersuchung über die Lage der Presse in Lateinamerika bestellt. Parallel dazu wurde auch die Lage der Funkmedien studiert. Das Ergebnis war der im „Adveniat“-Hirtenbrief 1964 bekanntgegebene Beschluß, die Massenmedien in Lateinamerika ihrer Bedeutung wegen zu fördern.

Der Weltkongreß der katholischen Presse in New York machte den ersten konkreten Vorstoß zu einer großräumigen Planung. In der erwähnten Resolution wurde verlangt, daß „die Hilfe für die katholischen Massenmedien nicht der Willkür und dem Zufall persönlicher Verbindungen überlassen werden dürfe. Sie muß vielmehr mit Planung und Weitsicht wirksam werden. Dementsprechend sollten die Hilfsmaßnahmen aller Länder koordiniert und abgestimmt sein". Der Resolution folgte bald ein konkreter Akt: Ein Jahr später wurde eine eigene Entwicklungskommission der UCIP gegründet. Sie sollte die Koordinierung und Kooperation in der Entwicklungsarbeit und alles, was damit zusammenhängt, in Angriff nehmen.

Neben den Erfahrungen in der Aktion "Adveniat", die Gabel zu ihrem Konsulenten bestellt hatte, brachte eine Einzelaktion der Wochenzeitung im Bistum Essen, „Rubrwort", Erkenntnisse, wie eine Massenmedienhilfe in der Kirche heute aussehen müßte: Unter den Projekten der „Rubrwort ${ }^{\alpha}$-Aktion „Der Groschen macht's" (Ergebnis: in über drei Jahren ca. DM 470000 ) sind auch Strukturuntersuchungen über die Möglichkeiten eines Aufbaus einer gesamtasiatischen Nachtrichtenagentur und Studien über die gleiche Problematik in Afrika.

Verhandlungen der UCIP-Entwicklungskommission mit dem Werk „Misereor" führten zu dem Beschluß der für "Misereor" zuständigen Bischofskommission im Juli vergangenen Jahres, daß in Zukunft „Misereor" generell die Massenmedien fördern solle. Bislang hatte "Misereor" nur gelegentlich geholfen, wenn Massenmedien direkt dem Kampf gegen Hunger und Krankheit dienten. Vorwiegend sind dies „Radioschulen", die der Alphabetisierung dienen. Immerhin verlangen die Geldbeträge, die für solche $Z$ wecke eingesetzt worden sind, Respekt.

So hat die Zentralstelle für Entwicklungshilfe e. V. - sie verteilt Mittel aus dem Budget der deutschen Bundesregierung, die für Entwicklungsprojekte in kirchlicher Verantwortung bestimmt sind - über 21 Millionen DM investiert. Den Hauptteil davon nahm der Bau des Senders "Veritas" in Manila in Anspruch, der allerdings noch nicht funktioniert, weil man sich zu wenig mit der Frage beschäftigt hatte, woher das Geld für den laufenden Betrieb kommen solle. „Misereor" hat bisher über 11 Millionen DM aufgewendet, hauptsächlich für Rundfunkprojekte, die sich der Alphabetisierung widmen. Die Aktion "Adveniat" - allein für Lateinamerika tätig unterstïtzte jedoch ohne Beschränkung auf besondere Zwecke grundsätzlich alle Massenmedien und dotierte sie von 1964 bis heute mit über 7,5 Millionen DM. Davon sind allein für die Modernisierung von Agenturen, Zeitungen und Zeitschriften fast 4,5 Millionen DM aufgewendet worden. 
Mit entsprechend kleineren Beträgen hat sich das österreichische Hilfswerk „Fastenopfer" vor allem um den Ausbau der Presse in Korea bemüht. Das französische "Comité Catholique contre la Faim et pour le Développement" gab mehrere Hilfen in Höhe von 45000 DM. Auch das "Schweizer Fastenopfer" hat die Massenmedienhilfe in sein Programm aufgenommen. Die Niederlande haben kleinere Einzelprojekte finanziert. Über die zahlreichen privaten kleinen und großen Medienhilfen der Katholiken der USA gibt es keinen Gesamtüberblick, da nur unzulängliche Einzelinformationen vorhanden sind. Das neu gegründete kanadische Hilfswerk „Canada Aid“ ist ebenfalls zur Massenmedienhilfe bereit. Last not least sind noch die mannigfaltigen Hilfen zu erwähnen, die von der Propaganda Fide-Kongregation und den einzelnen nationalen Missionswerken gegeben wurden. Von den großen Aktionen sei der Bau einer Druckerei in Umtata angeführt, die das Päpstliche Missionswerk der Kinder in Deutschland mit DM 300000 ermöglichte.

Hieß vor einigen Jahren das Problem noch: Woher können wir Geld für die Massenmedien bekommen, so heißt das Problem heute: Wohin und wem soll das Geld gegeben werden? Es hat sich nämlich in den Kontinenten rasch herumgesprochen, daß Massenmedien auf der katholischen Entwicklungsbörse in Kurs kommen. So nimmt die Flut von Hilfsanträgen, die Zahl von Besuchern, die Geld für den Aufbau neuer Massenmedien haben wollen, von Tag zu Tag zu. Die Geldmittel sind aber nicht unbeschränkt. Wo und wie soll nun geholfen werden?

Die einzelnen Hilfswerke arbeiten nur "projektgebunden". Sie beschäftigen sich jeweils mit einem konkreten Projekt, das danach beurteilt wird, ob es innerlich solide und entwicklungspolitisch richtig ist. Planung, sei sie räumlich oder medienstrategisch, ist jedoch nicht Aufgabe der Hilfswerke. Dies ist ihr Standpunkt. Bei CIDSE, der "Coopération Internationale pour le Développement Socio-Economique“, zu der sich die europäischen Hilfswerke in Brüssel zusammengeschlossen haben, wird eine Zentralkartei eingerichtet. In dieser sollen alle bei den Hilfswerken eingehenden Projekte registriert werden. Jedes Hilfswerk kann daraus erfahren, welche Institution sich mit welchem Projekt befaßt. Auf diese Weise wird eine Doppelbearbeitung und eine mögliche Doppelsubventionierung vermieden.

Diese Massenmedienhilfe in der dargelegten Form der Projektbearbeitung ist ein gewaltiger Fortschritt, weil so auf jeden Fall einmal geholfen wird. Dennoch darf aber ihre problematische Kehrseite nicht übersehen werden. Denn die derzeitige, gleichsam "atomisierte" Art der Massenmedienförderung, so gut gemeint sie auch sein mag, kann zu einer mondialen Fehlentwicklung und Fehlsteuerung der katholischen Massenmedien führen. Gewaltige Geldmittel können so vergeudet werden. Das Ende würden Enttäuschung und Resignation sein.

Warum lernen wir nicht von der Welt? Sie ist sichtbar auf dem Weg zur Einheit. Von der Technik - denken wir hier an die Kommunikationsformen der Information und des Verkehrs - kann man dies zweifellos sagen. Wie steht es aber mit der Kirche? Ist sie nicht bereits, wie wir es im Credo formulieren, eine Welt-Einheit? Die universale, die "Katholon"-Kirche? Sie ist hierarchisch gegliedert, hat eben erst beim Konzil ihr weltweites Zusammenwirken sichtbar bewiesen und hat durch die klar formulierte Kollegialität der Bischöfe sogar die wechselseitige Verantwortung als Maxime der Kirche ins Bewußtsein gehoben.

Merkt man aber irgendwo diese Welt-Einheit der Kirche im Bereich ihrer Massenmedien? Nirgends. Vielleicht liegt ein Grund für dieses Versagen darin, daß bis vor kurzem noch die Kirche in den Massenmedien mehr die Gefahr und damit Anlaß zu Skepsis und Zurückhaltung gesehen hat als die Chance, ihr Tor zur Welt und zu den Menschen weit aufzustoßen. Leider scheint die Kirche - von den rühmlichen 
Ausnahmen abgesehen - auch heute noch nicht begriffen zu haben, was die Massenmedien in der Welt von heute bedeuten. Sonst müßte sie das Steuer herumreißen. Sonst dürfte sie nicht, wenn sie die Rangfolge ihrer Aufgaben erstellt, zuerst an Priesterseminare (wie es in Lateinamerika der Fall war), an Schulen, Hospitäler usw. denken - sie müßte vielmehr mit an die Spitze dieser Prioritätenlisten die Massenmedien setzen.

Wenn aber die Bemühungen um eine planvolle Massenmedienförderung Erfolg haben sollen, müssen zwei Kriterien in der Kirche Allgemeingut werden:

1. Die Massenmedien sind nicht "unchristlich“. Sie sind Medien, Mittel, die so schlecht und so gut sind, wie die Menschen schlecht und gut sind, die sich ihrer bedienen und die sie bedienen.

2. Bei den Massenmedien ist Sachkenntnis Voraussetzung, um mitreden und mitentscheiden zu können. Der Journalismus ist ein Beruf, den man wie jeden anderen mühsam erlernen muß.

Gesetzt den Fall, diese beiden - im säkularen Raum so selbstverständlichen Kriterien wären allgemein angenommen, wäre dann schon alles in Ordnung? Mitnichten. Denn die eigentliche Aufgabe, die sich uns stellt, heißt: Die Kirche als weltweite Institution muß weltweit planen. Sie kann den Aufbau und den Ausbau der Massenmedien nicht dem Freistil und Zufall überlassen.

Der Zufall, nach dem Projekte bei Hilfswerken eingereicht und finanziert werden, darf nicht der Maßstab für die Massenmedienhilfe bleiben. Die einzelnen Werke selbst können und wollen nichts anderes tun, als sich mit den ihnen zugegangenen Projekten beschäftigen. Es muß sich also eine andere Institution um Planung und Zusammenarbeit kümmern. Diese Aufgabe drängt mehr denn je. Einige Beispiele mögen dies illustrieren:

In einer Region haben Orden bereits Druckereien eingerichtet und geben Zeitungen heraus. Nun werden auch Bischöfe initiativ. Auch sie wollen Druckereien gründen, Zeitungen herausgeben usw. Das Ergebnis ist: Rivalität. Denn die einen wollen Geld zum Ausbau ihrer bereits bestehenden Anlagen, die anderen wollen Geld für „ihre“ neu geplante Druckerei und Subventionen für neue Zeitungen. Was tun? Alle Wünsche zu erfüllen ist jedenfalls sinnlos.

Oder: In einer afrikanischen Hauptstadt soll für eine bestehende Zeitung eine eigene Druckerei errichtet werden. Man braucht aber nicht nur Geld, sondern auch Drucker und Setzer. Wenn schon das Geld aufgebracht werden kann, woher sollen die Fachkräfte kommen?

Oder: Ein brasilianischer Bischof will drei Rundfunkjournalisten ausbilden lassen vielleicht für einen eigenen Lokalsender, den er noch plant, oder weil er Möglichkeiten für einen anderen Einsatz sieht. Sicher wird aber nicht nur er vor diesem Problem stehen. Es werden in Brasilien auch anderswo katholische Rundfunkjournalisten gesucht. Sollte da nicht zunächst untersucht werden, wie und wo eine ganz Brasilien umfassende Ausbildungsaktion gestartet werden kann?

An all diesen einfachen und doch so schwer zu lösenden Problemen zeigt sich nur wieder, wie notwendig strategische Planung in der Massenmedienhilfe ist. Doch: Wer soll planen? - Wer soll Untersuchungen machen? - Wer soll daraufhin Initiativen entwickeln? - Wer soll Fachleute als Berater und Ausbilder offerieren? Wer soll medienpolitische Abstimmungen zwischen Bischofskonferenzen und Orden anregen? - Wer soll die Orden untereinander zur Zusammenarbeit gewinnen? Diese Aufgaben warten auf eine Institution, die sich ihrer annimmt.

Die Entwicklungskommission der UCIP ist ein erster Ansatz für eine solche, vom Papst überantwortete Planungs- und Koordinierungsfunktion. Leider gibt es inner- 
halb der UNDA und der OCIC, den beiden anderen katholischen Weltorganisationen für Funk und Film, noch keine ähnliche Initiative. Und doch müssen Presse, Funk, Fernsehen und Film in einer weltweiten strategischen Massenmedienkonzeption als Einheit gesehen und behandelt werden.

Grundsätzlich wäre für diese Aufgabe einer weltweiten Koordinierung der Massenmedien die Päpstliche Kommission für die Massenmedien zuständig. Es besteht auch bei anderen wichtigen Stellen in Rom (Staatssekretariat und PWG) größtes Interesse an einer planmäßigen Förderung der Massenmedien. Angesichts der Fachproblematik wie auch gewisser kircheninterner Schwierigkeiten, die nicht übersehen werden dürfen, ist aber die Frage nicht bloß rhetorisch, ob die Päpstliche Kommission für die Massenmedien eine solche Initiative, d. h. dynamische und anpassungsfähige Institution sein kann, die vorhin geschilderten Aufgaben zu bewältigen. Die Päpstliche Kommission kann viel mehr Gutes tun, wenn sie den für Planung, Kooperation und Koordination arbeitenden Kräften subsidiär ihre Hilfe und Unterstützung zuteil werden läßt. Wenn auf diesem Gebiet, zumal da den katholischen Massenmedien in den Entwicklungsländern das Wasser bereits bis zum Hals steht, alle, die etwas tun können, ihr Helfen-Wollen höher ansetzen als ihr Prestige, dann könnte sich eines Tages aus der Praxis heraus ein Institut der Medienplanung und -kooperation entwickelt haben, gleichsam ein imaginärer „runder Tisch" für alle, die sich mit der konkreten Entwicklungsarbeit für die Massenmedien beschäftigen.

$\mathrm{Zu}$ den Aufgaben eines solchen Instituts würden gehören:

1. Es gilt, Fachleute aus allen Medienbereichen und deren Sparten in einer Personalkartei zu exfassen, damit jederzeit und für jedes Projekt fachlicher Rat eingeholt werden kann.

2. Es gilt, die kompetenten Vertreter der auf dem Gebiet der Massenmedien tätigen Orden zu gewinnen. Einen schon erfreulichen Ansatz zu einer solchen Zusammenarbeit - nicht allein bei den Massenmedien - gibt es in SEDOS. Unter dem unscheinbaren Namen "Servizio di Documentazione e Studi“ haben sich bis jetzt rund 30 männliche und weibliche Orden und Genossenschaften zusammengeschlossen, um - ich zitiere aus der schriftlichen Zielsetzung - „in gemeinsamem Bemühen die Missionsaufgabe der Kirche wirksamer erfüllen zu können". Aus einigen Namen kann man die Kompetenz für Massenmedienfragen erkennen: Jesuiten, SVD, Oblaten (OMI), Weiße Väter, Mary Knoll Fathers und andere.

3. Es gilt, regionale und auch kontinentale Auf- und Ausbaupläne zu erarbeiten. Hierbei müssen die Interessen der Orden wie der lokalen Kirchen koordiniert werden. Entscheidend für die Priorität der Aufgaben muß sein, wie notwendig und wie effizient sie sind. Wo immer in den Massenmedien Laien initiativ tätig sind, sollten diese - gemäß dem ihnen zukommenden "Weltamt" - katalysierend in der Entwicklungskonzeption gesehen werden.

4. Es gilt, nicht nur die finanzielle Bedrängnis der katholischen Massenmedien zu sehen - noch ernster vielleicht sind die personellen Schwierigkeiten. Wir haben zu wenig ausgebildete Redakteure, zu wenig Fachleute in der Technik und Verwaltung.

5. Vielleicht kann eine solche Initiative der Koordination und Kooperation auch eine neue missionarische Genossenschaft auf den Plan rufen, deren Aufgabe die einer „Massenmedien-Feuerwehr" sein würde. Ihre Mitglieder müßten bestbefähigte und ausgebildete Journalisten, Druckereifachleute, Rundfunk- und Fernsehtechniker, Studioleiter, Kameramänner und Regisseure sein. Sie könnten hier für drei Jahre eine darniederliegende Druckerei in Schwung bringen, dort eine lokale Nachrichtenagentur gründen, anderswo wieder ein Rundfunkstudio einrichten oder als Lehrer in einer Journalistenschule tätig werden. Gewiß gibt es schon ausgebildete Fachleute in ein- 
zelnen Genossenschaften. Aber wenn man früher Orden für die Erziehung in Schulen gegründet hat, warum sollte es nicht heute Genossenschaften geben, seien sie wie immer auch strukturiert, die sich nur den Massenmedien widmen?

6. Es gilt auch, die gemeinsame Arbeit mit nichtkatholischen Gruppen zu planen. Wie viele Energien könnten im Zeichen einer solchen Okumene frei werden, um anderen nötigen Aufgaben zugeführt zu werden?

Welche Gestalt auch immer eine solche Aktion organisatorisch und juridisch haben mag - als Arbeitsgemeinschaft, als Institut - stets wird sie den Charakter des Dienstes haben müssen. Sie kann keine Kommandozentrale, auch keine Stelle, wo Geld verteilt oder versprochen wird, sein. Sie soll aber durch ihr unablässiges Bemühen um sachlichen Interessenausgleich und durch detaillierte Planungsarbeit helfen, daß die vorhandenen Geldmittel nicht mehr nach dem zufälligen Bedarf oder nach dem Gießkannenprinzip, sondern nach den Erfordernissen einer auf die Zukunft hin orientierten Schwerpunktkonzeption verteilt werden.

Solche Gedanken sind nicht utopisch, sondern im Gegenteil, höchst real. Tut nämlich die Kirche nichts, verschließt sie sich der Notwendigkeit dieser Planung ihrer Massenmedien, dann kapituliert sie vor dem Auftrag Christi: euntes docete!

Diesen Auftrag kann sie heute aber nur mehr mit Hilfe der Massenmedien erfüllen. Darum muß einfach die Kirche mit dem ernst machen, was ihr der heilige Lukas über Planung ins Stammbuch geschrieben hat. Die Prinzipien für ein solches Massenmedienkonzept sind ebenso einfach:

1. Das Beste ist gerade gut genug.

2. Im Heute verhält sich richtig, wer gestern schon an das Morgen gedacht hat.

\section{S U M M A R Y}

The Catholic Church, represented by its institutions and organisations, by the Council and the Pope, wants an additional aid for mass media in the developing countries. At present many pertinent actions are already under way. The German collecting campaigns "Misereor" and "Adveniat" have spent more than 11 respectively 7 millions DM for this action until today. Catholic activities in the USA, Austria, Switzerland, the Netherlands and France, also the Roman Curia, have given similar aid. A step forward is the arrangement of a central registration for the individual actions: by the "Coopération Internationale pour le Développement Socio-Economique" (CIDSE) in Brussels. However the "atomistic" kind of actions remains quite a problem, until there is no practical coordination of developing programs. For different historical reasons the Church as a worldwide institution was standing somewhat in the background until now. Occasionally regional progress brought differences and caused rivalries. The establishment of a central institute for media-planning and media-cooperation: i. e. a "round table" for people engaged in developing the mass media will be necessary. This institute should not be identical with the Papal Commission for the Mass Media.

\section{RESUMEN}

La iglesia católica representata por sus sociedades e organisaciones, por el Concilio y el Papa desea una ayuda mas intensiva para los medios de comunicación en los paises subdesarollados. Por el momento ya se aplican numerosas medidas de ayuda. Las Obras de Cooperación alemanas "Misereor" e "Adveniat" aplicaron con este motivo 11 o mas de 7 milliones de Marcos respectivamente. Las obras caritativas católicas en USA, Austria, 
Suiza, Holanda, Françia y también los departamentos de la Curia romana han dedicado cada uno por su parte la correspondiente ayuda. Ahora se organiza por lo menos un Tarjetero Central sobre cada una de las medidas en la "Coopération Internationale pour le Développement Socio-Economique" (CIDSE) en Bruselas. La desorgánica forma de esta promoción permanece problemática en tanto no halla una planificación y realización coordinada. La iglesia como organización estendida por todo el mundo se ha mantenido hasta ahora por diversos razones históricos en la retaguardia. Avances locales llevan a veces arivalidades. Es neçesario la erección de un Instituto Central para planificación y cooperación e una "mesa redonda" para todos los que se ocupen de la ayuda al desarollo de los medios de comunicación. El instituto debería sin embargo no ser idéntico con la Comisión Pontificial para medios de comunicación.

\title{
Der Welttag der Kommunikationsmittel als Instrument kritischer Bewußtseinsbildung
}

\author{
von Walther Kampe
}

Das Konzilsdekret über die gesellschaftlichen Kommunikationsmittel sieht in Nr. 18 vor, daß in allen Diözesen des Erdkreises jährlich ein Tag festgesetzt werde, an dem die Gläubigen auf ihre Aufgaben in den Bereichen von Presse, Film und Funk aufmerksam gemacht werden. Dabei sollen sie auch im Gebet der Menschen gedenken, die besondere Verantwortung auf dem Feld der Publizistik tragen. Ferner regt das Dekret an, daß die Gemeinden eingeladen werden, durch finanzielle Unterstützung dazu beizutragen, daß die publizistischen Einrichtungen der Kirche den Anforderungen der Zeit entsprechen. In dem vorhergehenden Abschnitt des Dekrets war bereits gesagt worden, es sei der Kirche unwürdig, daß ihre Glieder untätig zusehen müssen, wenn das "Wort des Heiles" durch technische Unzulänglichkeiten und ungenügende Mittel behindert werde. Es ist ungewöhnlich, aber doch realistisch, daß ein Konzilsdokument sich mit Finanz- und Organisationsfragen befaßt, denn ohne eine gesunde wirtschaftliche Basis sind zumal in der heutigen gesellschaftlichen Situation alle gutgemeinten Anstrengungen auf dem publizistischen Gebiet zum Scheitern verurteilt.

Walther Kampe, Weihbischof der Diözese Limburg/Lahn, leitet das Hörfunk- und Fernsehreferat innerhalb der Publizistischen Kommission der Deutschen Bischofskonferenz. Während des II. Vatikanischen Konzils sorgte er für eine umfassende Information der Journalisten aus dem deutschen Sprachraum und trug wesentlich zur Unterrichtung der deutschen Offentlichkeit über die Beratungen und Entscheidungen der Kirchenversammlung bei. Im Auftrag der deutschen Bischöfe leitete er die Vorbereitungen zum diesjährigen "Welttag der Kommunikationsmittel" (26. Mai). Der vorliegende Beitrag weist über diesen konkreten Anlaß hinaus auf die notwendigen Aufgaben kirchlicher Offentlichkeitsarbeit in der Zukunft. 